\title{
A Central Symmetrical and Low-Profile Omnidirectional Circularly Polarized Antenna
}

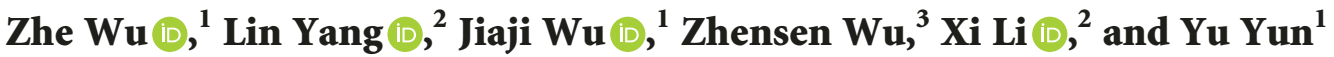 \\ ${ }^{1}$ The School of Electronic Engineering, Xidian University, Xi'an 710071, China \\ ${ }^{2}$ National Key Laboratory of Antennas and Microwave Technology, Xidian University, Xi'an 710071, China \\ ${ }^{3}$ The School of Physics and Optoelectronic Engineering, Xidian University, Xi'an 710071, China
}

Correspondence should be addressed to Lin Yang; lyang@mail.xidian.edu.cn

Received 21 May 2019; Accepted 30 July 2019; Published 24 September 2019

Academic Editor: Giuseppina Monti

Copyright (c) 2019 Zhe Wu et al. This is an open access article distributed under the Creative Commons Attribution License, which permits unrestricted use, distribution, and reproduction in any medium, provided the original work is properly cited.

\begin{abstract}
In this paper, a compact circularly polarized (CP) antenna with omnidirectional radiation is presented, which has a central symmetric configuration (CSC) and planar structure. It consists of three identical end-fire CP antenna units. Based on the combination of two parallel complementary current sources, each unit cell can be realized by a scalloped cavity and a couple of curved strip lines. Due to a single-layer circular structure with low profile, this configuration can be excited by a coaxial probe in the centre of the whole frame simultaneously. In addition, this prototype was miniaturized with a diameter of $32 \mathrm{~mm}\left(0.266 \lambda_{r}\right.$, the wavelength of the centre frequency in free space is represented by $\left.\lambda_{r}\right)$ and a height of $1.5 \mathrm{~mm}\left(0.012 \lambda_{r}\right)$. Centre resonance frequency $2.492 \mathrm{GHz}$ is selected, resulting in an omnidirectional radiation and impedance bandwidth (VSWR $\leq 2)$ of $25 \mathrm{MHz}(2481-2506 \mathrm{MHz})$.
\end{abstract}

\section{Introduction}

Since wireless communication systems have developed fast in recent years, the $\mathrm{CP}$ antenna has attracted strong attention due to its advantage of anti-multipath interference. Particularly, owing to 360 degree coverage and the same radiation strengths of the CP antenna, the omnidirectional CP antenna becomes very attractive. Therefore, a great deal of effort has been directed to design omnidirectional CP antennas [1-11].

Lots of classical types of antennas have been designed for omnidirectional CP antennas recently. In the approach in [1], this was attained by dielectric resonator antennas (DRAs). It excited a degenerate mode for a CP antenna by introducing some slots to the side surfaces of the DRA. To give a good AR, the slots should be designed correctly for perturbation of circular polarization. Similarly, the impedance is adjusted by the probe length to match according to the principle of antenna. Additionally, with the presence of the associated feeding network, four bended monopoles were excited simultaneously [3]. This antenna has a working bandwidth $(\mathrm{S} 11 \leq-10 \mathrm{~dB}$ and $\mathrm{AR} \leq 3 \mathrm{~dB})$ of $87 \mathrm{MHz}$, and electrical size is about $0.22 \lambda_{0} \times 0.22 \lambda_{0} \times 0.076 \lambda_{0}$. It has a small transverse size but a slightly high profile. $\mathrm{CP}$ radiation is obtained by a monopole and spoke-like metal loop strips [5], the electric monopole is acted by a coaxial probe and the loop plays the part of the magnetic monopole. To achieve an electric monopole, this design introduces double layers for appropriate probe length (about $0.079 \lambda_{0}$ ). Besides, four outer arcs and an inner disk-loaded feeding pin can generate horizontal and vertical polarization, respectively, in [7]. For good impedance matching, an additional lower substrate is necessary for matching network to be arranged. However, all these achievements have nonplanar or multilayered structures, and their electrical profiles are relatively large obviously.

Certainly, several single-layer designs have also been put forward to realize the omnidirectional CP. One of the examples is given in [4], where it is based on a vertical polarization produced by a zeroth-order resonance (ZOR) mode of epsilon negative (ENG) transmission line (TL) and a horizontal polarization generated through the curved branches. By placing four curved zeroth-order resonators (EZR) and mu-zero resonance (MZR) resonators in a circle loop form array [11], the MZR and EZR operate as magnetic dipole and electric dipole, respectively. Both of them have 
single-layer structure with low profiles, yet their whole dimensions need to be decreased.

There are also some new constructs with compact and low-profile feature of omnidirectional CP antenna. For example, in [2], the radiator of this antenna comprises a quarter-wave shorted patch and two printed strips, which can be considered the magnetic dipole and electric dipole, respectively. It has an average $\mathrm{AR}$ below $1.5 \mathrm{~dB}$ and a dimension of $1.74 \lambda_{0} \times 0.31 \lambda_{0}$. In [6], the design is based on several identical end-fire CP antenna units in a circular antenna array, and its overall dimension is $0.62 \lambda_{0} \times 0.62 \lambda_{0}$. There is no doubt that the aforementioned antennas all perform well, but the overall size of each of them needs to be further decreased to achieve miniaturization.

This paper presents a CSC and compact CP antenna with omnidirectional radiation motivated by aforementioned achievements. A planar circular array with the CSC composed of several end-fire CP antenna units is proposed. It has a compact single-layer structure with small size and a single feed and can be fabricated on a F4B easily (relative permittivity $\varepsilon_{r}=2.65$ with a thickness of $1.5 \mathrm{~mm}$ ). In both planar configurations, the proposed antenna has a wider usable bandwidth and a smaller size compared to that proposed in [4]. The working bandwidth of the antenna in [6] is larger than that of this antenna, but the proposed antenna is much smaller in size, realizing the miniaturization technology. The effective operation frequency band covers the whole $S$ band of BeiDou Navigation System with good directivity in the whole azimuth plane.

\section{Antenna Design}

2.1. Antenna Geometry and Planar Sector-Shaped Unit Element. In order to achieve an omnidirectional CP antenna with low profile and small size, we adopt a single-layer structure as much as possible. Certainly, the antenna must be designed to meet the antenna parameter requirements, such as VSWR, antenna gain, and AR. As for the omnidirectional antenna, it also needs to meet the requirements of out-of-roundness.
The geometry of the proposed CSC antenna based on a fan-shaped configuration that embraces three unit elements is presented in Figure 1. An element is composed of a scalloped cavity including one metallic hole and a couple of printed curved strips on each side of the board in the opposite azimuth direction. Two conductive curved strips are connected to a common scalloped cavity. One is connected to the upper left corner on the top, while the other to the lower right corner at the bottom of the unit element. In addition, double coupled lines and conductive curved strips have opposite directions on the same layer as is shown in Figure 1(d). The front and back structures are almost the same, but the coaxial radius is hollowed out at the bottom for welding.

2.2. Antenna Design Guideline. The original prototype structure is shown in Figure 2(a). When the feeding port is located near the shorted electric wall, the electric field can be excited in the open aperture. Since the free space wavelength is long enough compared with the profile of the dielectric substrate, the tangential electric field $\overrightarrow{\mathbf{E}}$ at the open aperture can be known as a magnetic current source $\overrightarrow{\mathbf{M}}_{\mathbf{s}}$ on the basis of the theory of boundary condition for electromagnetic fields:

$$
\overrightarrow{\mathbf{M}}_{\mathbf{s}}=-\overrightarrow{\mathbf{e}}_{\mathbf{n}} \times \overrightarrow{\mathbf{E}}
$$

where $\overrightarrow{\mathbf{E}}$ represents the tangential electric field of the radiation aperture excited in the state of dominate mode and the normal vector is symbolized by $\overrightarrow{\mathbf{e}}_{\mathbf{n}}$. The magnetic current is generated by the open aperture, whereas the double straight lines offer the electric currents. We are able to gain the electric far fields from [2]

$$
\begin{aligned}
& \vec{E}_{\theta}=-j k_{0} \frac{e^{-j k_{0} r}}{4 \pi r}\left(\eta_{0} f_{x} \cos \theta \cos \varphi-f_{m x} \sin \varphi\right), \\
& \vec{E} \varphi=j k_{0} \frac{e^{-j k_{0} r}}{4 \pi r}\left(\eta_{0} f_{x} \sin \varphi-f_{m x} \cos \theta \cos \varphi\right),
\end{aligned}
$$

where $\eta_{0}=120 \pi$ and

$$
\begin{aligned}
f_{m x}= & \int_{-l_{m} / 2}^{l_{m} / 2} \vec{M}_{x}\left(x^{\prime}, y^{\prime}, z^{\prime}\right) e^{j k_{0} x^{\prime} \sin \theta \cos \varphi} d x^{\prime} \\
= & \vec{A} l_{m} \sin c\left(\frac{l_{m}}{2} k_{0} \sin \theta \cos \theta\right), \\
f_{x}= & \int_{-l_{e}-\left(l_{m} / 2\right)}^{-\left(l_{m} / 2\right)} \vec{J}_{x 1}\left(x^{\prime}, y^{\prime}, z^{\prime}\right) e^{j k_{0} x^{\prime} \sin \theta \cos \varphi} d x^{\prime} \\
& +\int_{l_{m} / 2}^{l_{e}+\left(l_{m} / 2\right)} \vec{J}_{x 2}\left(x^{\prime}, y^{\prime}, z^{\prime}\right) e^{j k_{0} x^{\prime} \sin \theta \cos \varphi} d x^{\prime} \\
= & 2 \vec{B}^{j(\pi / 2)} l_{e} \frac{\pi}{\pi^{2}-\left(k_{0} l_{e} \sin \theta \cos \varphi\right)^{2}}\left\{\cos \left[k_{0}\left(l_{e}+\frac{l_{m}}{2}\right) \sin \theta \cos \varphi\right]+\cos \left(k_{0} \frac{l_{m}}{2} \sin \theta \cos \varphi\right)\right\},
\end{aligned}
$$




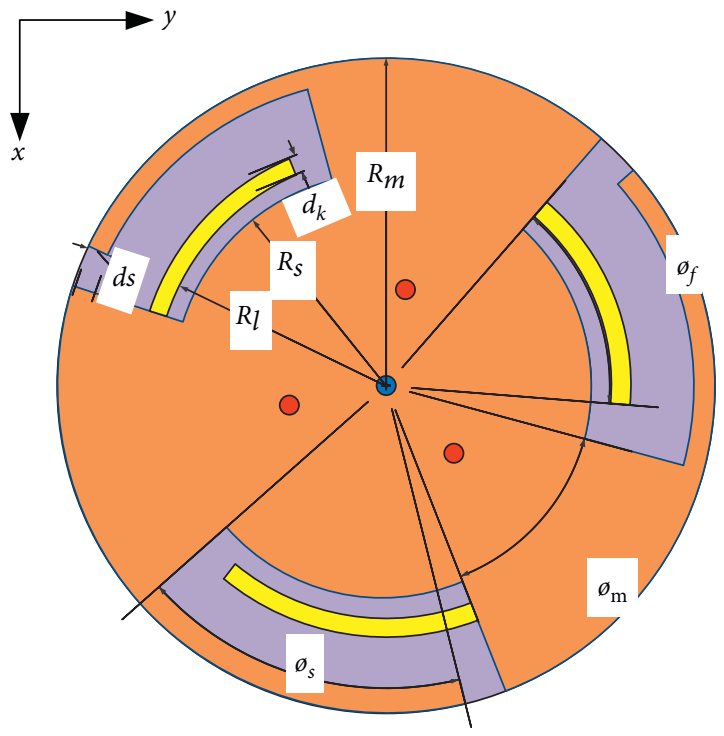

(a)

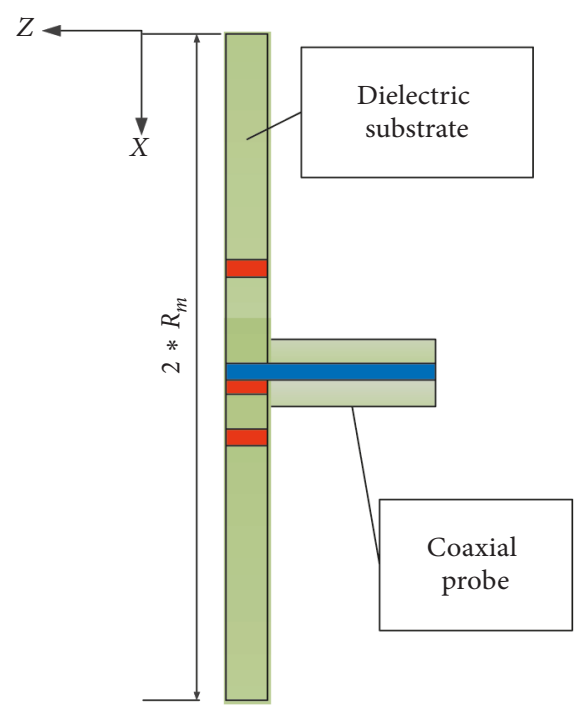

(c)

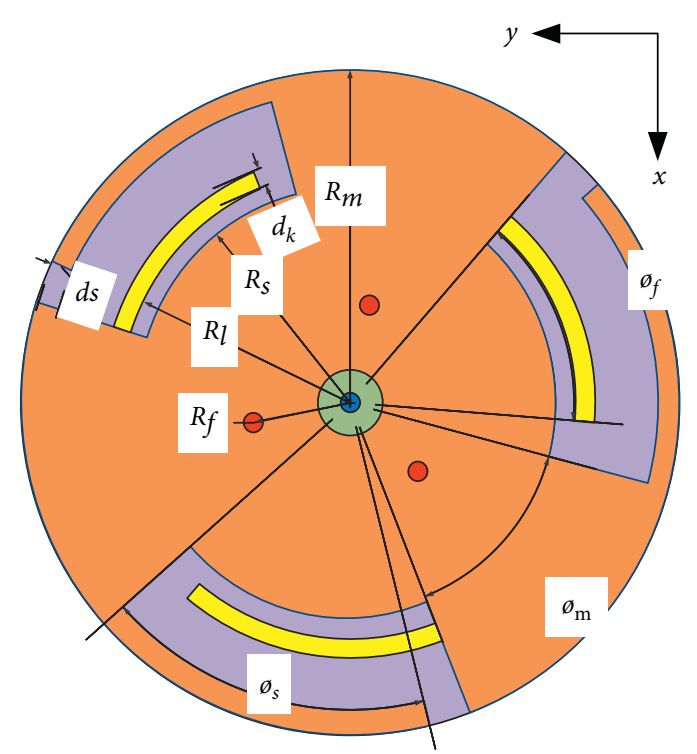

(b)

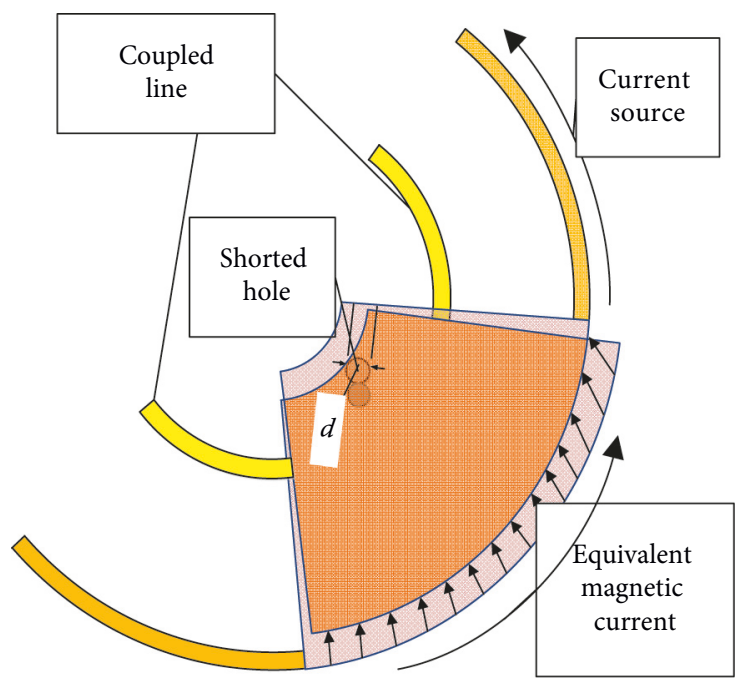

(d)

Figure 1: Geometry of the proposed omnidirectional CP antenna: (a) top layer; (b) bottom layer; (c) side view; (d) unit element.

where $\vec{M}_{x}\left(x^{\prime}, y^{\prime}, z^{\prime}\right), \vec{J}_{x 1}\left(x^{\prime}, y^{\prime}, z^{\prime}\right)$, and $\vec{J}_{x 2}\left(x^{\prime}, y^{\prime}, z^{\prime}\right)$ are magnetic current and electric current distributions, while $\vec{A}$ and $\vec{B}$ are the magnetic current and electric current, respectively. The far-field $\vec{E}_{\theta}$ and $\vec{E} \varphi$ are obtained by the magnetic current and electric current, respectively. When the lengths of the complementary dipoles are controlled reasonably, $\vec{E}_{\theta}$ and $\vec{E} \varphi$ with equal magnitude in the yoz plane can be obtained.

It is known from [2] that when the electric field intensity is maximum (the peak magnetic current is realized), the surface electric current, however, reaches the minimum. After a quarter of a period, the condition is just opposite. It reveals that the magnetic current has a $90^{\circ}$ phase difference from the electric current. With the inherent $90^{\circ}$ phase difference between the two types of currents, a circular polarization radiation pattern can be achieved. The linear parallel complementary dipoles were converted into those annulus ones in this work shown in Figure 1(d). Therefore, the magnetic current has an inherent phase difference of $90^{\circ}$ from the electric currents in the proposed CSC antenna. This conclusion has been verified in Figures 2(b) and $2(\mathrm{c})$.

Inspired by the aforementioned CP mechanism, a CSC circular array comprising three identical end-fire CP elements is proposed in this paper. A shorted scalloped construct is formed by introducing a metallic aperture along the angular bisector. When several structures are placed with the CSC as in the proposed design, partial radiation will be cancelled out from their side apertures. Therefore, the effective radiation is only from the arcshaped one. The electric current and magnetic current parallel to each other were converted into annular current source at the same time in the final design. 


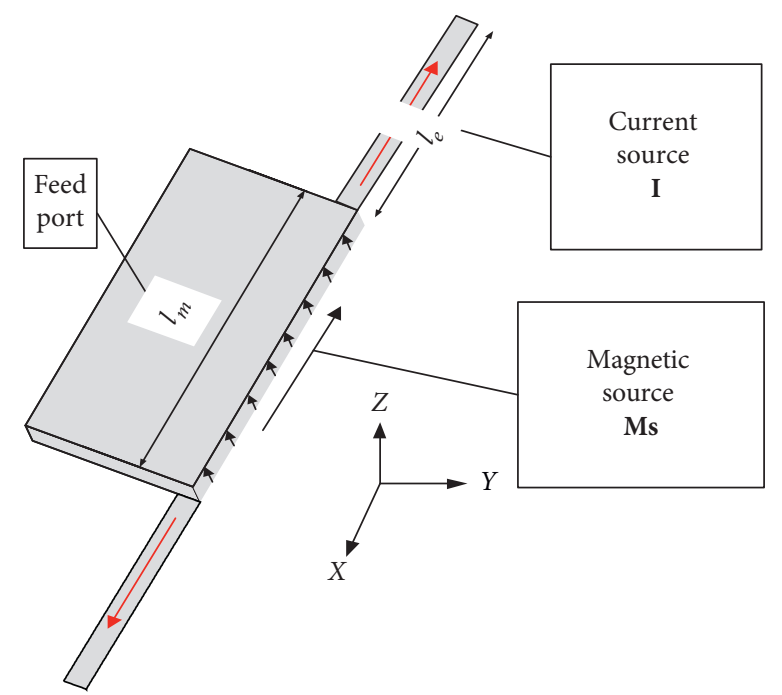

(a)
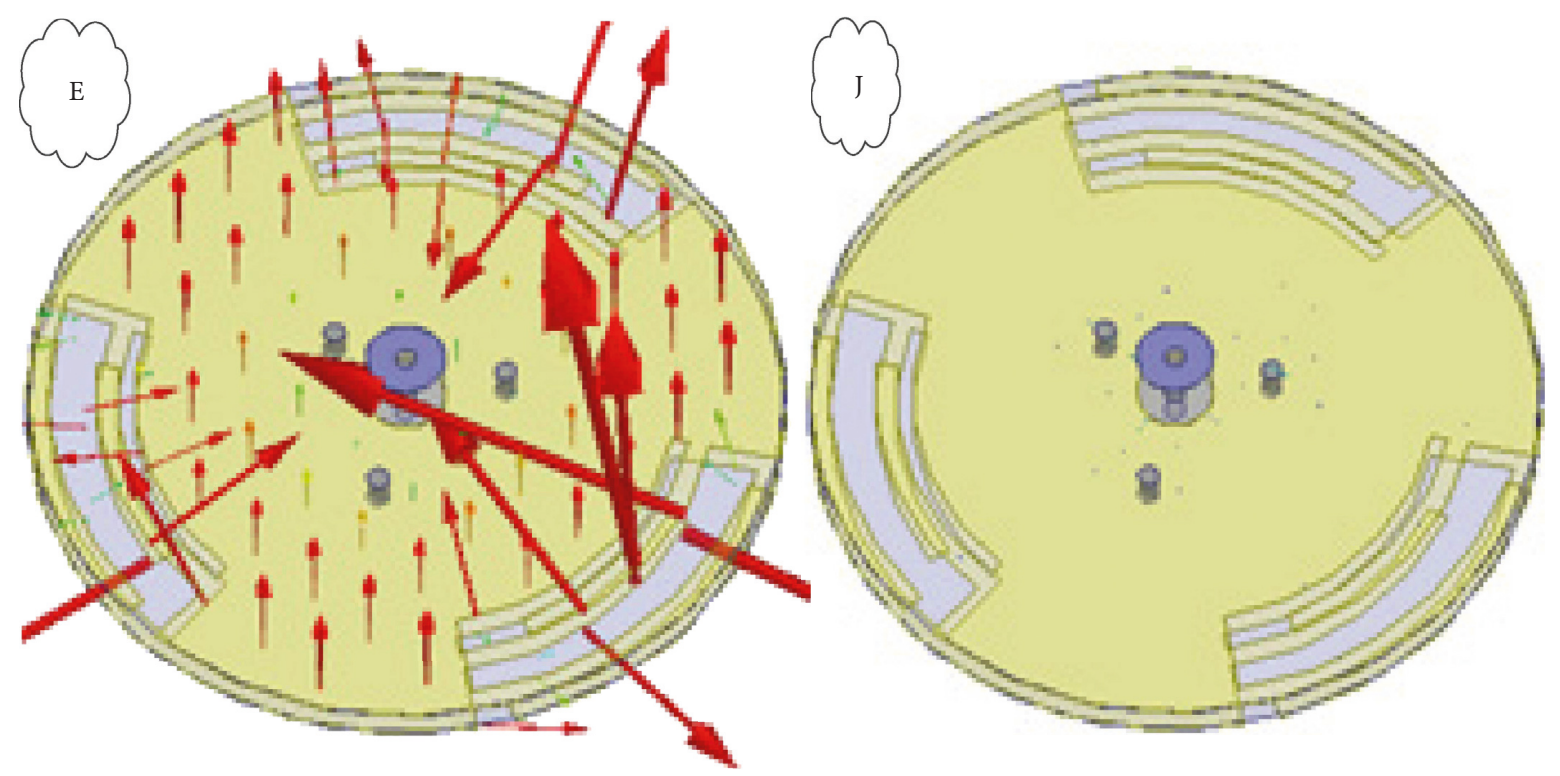

(b)

Figure 2: Continued. 

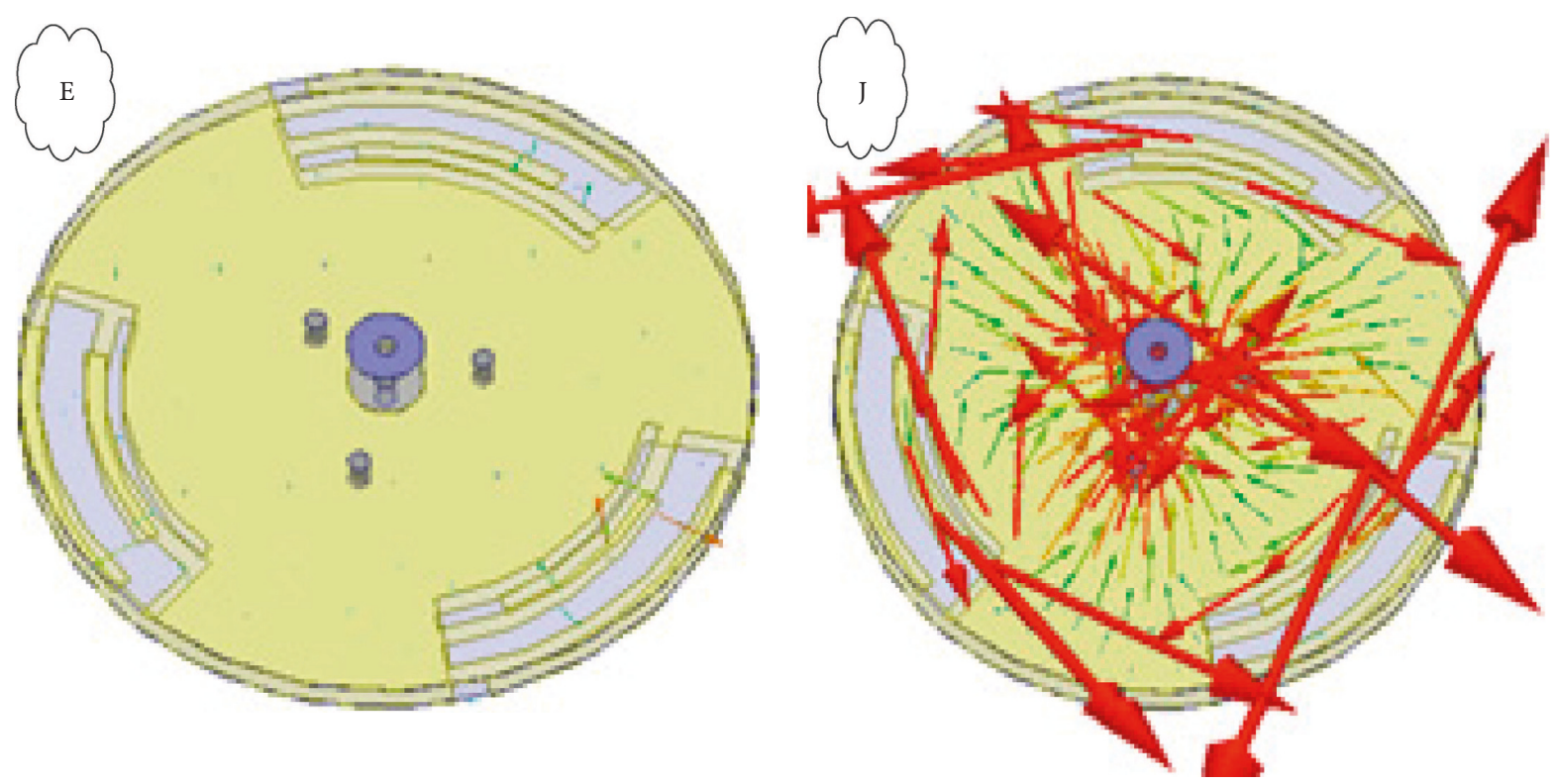

(c)

Figure 2: Sectorial cavity unit prototype structure (a), electric-field distribution E and surface electric current distribution J for the proposed antenna (b) $t=0$. (c) $t=T / 4$.

According to the classical electromagnetic field theory, the relationship between the radius of the circular patch and the resonant frequency can be determined by using the following equations:

$$
\begin{aligned}
f_{n p} & =\frac{X_{n p}^{\prime} c}{2 \pi a_{\mathrm{e}} \sqrt{\varepsilon_{r}}}, \\
a_{\mathrm{e}} & =a \sqrt{1+\frac{2 H}{\pi a \varepsilon_{r}}\left(\ln \frac{\pi a}{2 H}+1.7726\right)},
\end{aligned}
$$

where $X_{n p}^{\prime}$ is the zero-order term of the derivative of $n$-order Bessel function (for $\mathrm{TM}_{01}$ mode, $X_{01}^{\prime}=3.83171$ ) and parameters $a$ and $a_{\mathrm{e}}$ denote the physical radius and effective radius of the patch, respectively. In addition, the speed of light and the thickness of the substrate are represented by parameters $c$ and $H$, and $f_{n p}$ and $\varepsilon_{r}$ are corresponding to the resonant frequency and relative dielectric constant, respectively.

For a CSC antenna array in the xoy plane, the array factor can be expressed by

$$
\mathrm{AF}=\sum_{i=1}^{N} \alpha_{i} e^{j\left[k r \sin \theta \cos \left(\varphi-\varphi_{i}\right)+\beta_{i}\right]},
$$

where $\alpha_{i}$ and $\beta_{i}$ represent the amplitude and phase of the unit $i$, respectively. Parameter $r$ is the radius of the antenna array circle, and $\varphi_{i}=2 \pi i / N$. When the whole antenna's elements are excited with equal amplitude in phase, an omnidirectional radiation performance can be obtained in the azimuth plane. Based on the reason that too many units will cause the annular current to overlap and the theory that the omnidirectional performance requires at least three elements to be guaranteed, this paper chooses $i=3$ to meet the needs for the antenna design.

To clarify the concept of proposed antenna more clearly, the design process is demonstrated in Figures 3(a)-3(c). Figures 3(a) and 3(b) display the formation configuration of linear polarization and circular polarization, respectively. According to [9], the lowest mode (TM01mode) that could produce a monopole radiation pattern is resulted from conductive apertures shown in Figure 3(a). By introducing the curved strips, the CP mode is formed, thanks to the complementary dipoles presented in Figure 3(b). We now turn our attention to the most apparent merits-miniaturization-of this proposed design, demonstrated in Figure 3(c). With the cooperation of the coupling strips, the resonant frequency is largely reduced. The influence of the variation of specific variable parameters on the antenna performance is given in detail in Section 3.

The conception of this configuration is inspired from [6], both of them have the same CSC. Nonetheless, the formation of the $90^{\circ}$ phase difference is quite different. The $90^{\circ}$ phase difference in [6] is caused by the phase compensate line, yet the proposed antenna naturally exists. Above all, the proposed antenna is much smaller than that in [6] in dimension size, which shows miniaturization with compact structure.

The mechanism of the proposed antenna is similar to that in [2]. Since the linear complementary dipoles were converted into annular ones, it saves a lot of space with a quite compact structure. In addition, the resonant frequency is largely reduced by the shorting pins and coupling arcs. Therefore, the biggest advantage of the proposed antenna compared with that in [2] is its achievements in miniaturization.

As can be seen from a quarter-wave shorted patch in [2], the radius of the proposed antenna is comparable to a 




(a)

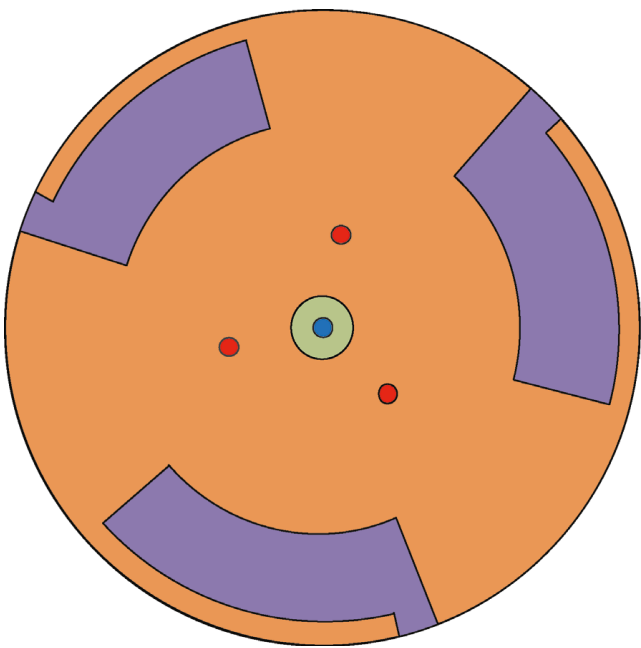

(b)

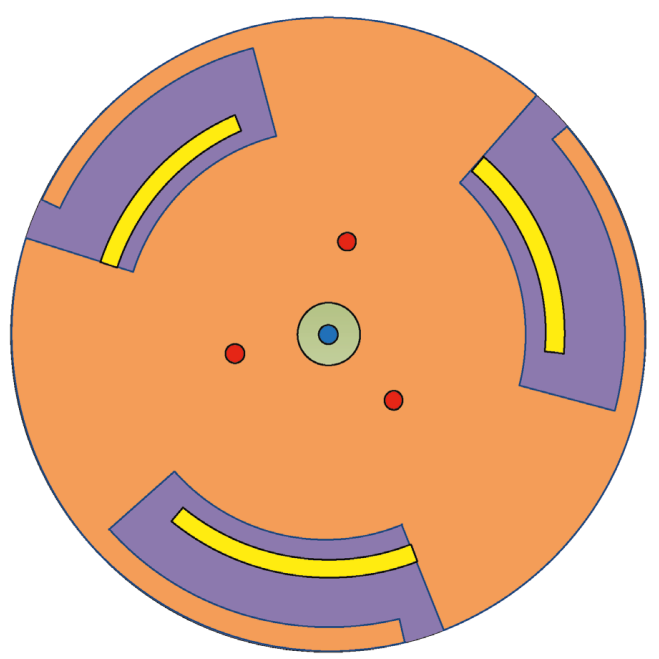

(c)

Figure 3: Evolution of the proposed antenna.

quarter working wavelength. However, the actual size is much smaller than the quarter working wavelength due to miniaturization technology. In the same way, the arc length of the complementary dipoles is also less than the quarter working wavelength to ensure the resonance of the ring current sources. Finally, we know that it is the coupling arcs and shorting pins that realize the miniaturization of the proposed antenna. Therefore, it is necessary to increase the length of the coupling arcs as much as possible to reduce the resonance frequency. As to the shorting pins, the closer it is to the centre of the circle, the lower the resonant frequency is. In combination with the antenna performance, various parameters are adjusted for optimization.

Figures 4(a) and 4(b) illustrate the results of a simulation including the magnitude of the electric field energy distribution and the surface current distribution on it, respectively. Meanwhile, Figures 4(c) and 4(d) show the LHCP and RHCP 3D-plot radiation pattern of the proposed CSC antenna. It can be found that energy is mainly concentrated in three sectors and coupled rings from Figures 4(a) and 4(b). As it can be seen from the 3D-plots of Figures 4(c) and 4(d), the proposed CSC antenna has good $360^{\circ}$ coverage performance and a low cross-polarization.

\section{Parametric Studies}

In order to better comprehend the mechanism of this proposed CSC antenna, we have investigated the effect of different related parameters on antenna performance. Several parameters, such as the coupling strips, the radius of the antenna, the position of the shorted aperture, and the length of the branches, were studied as described below.

3.1. Effect of Coupling Strips. It is the coupling strips that contribute to the miniaturization of this work to a large extent. The shorting pins act as several closed inductances, while the coupling strips can be considered as the closed capacitances. Hence, the closed inductances and capacitances can reduce the resonant frequency tremendously presented in Figure 5. It is perspicuous that the resonant frequency of the proposed antenna is near $3 \mathrm{GHz}$ without coupling ring, while about $2.45 \mathrm{GHz}$ with coupling ring. 


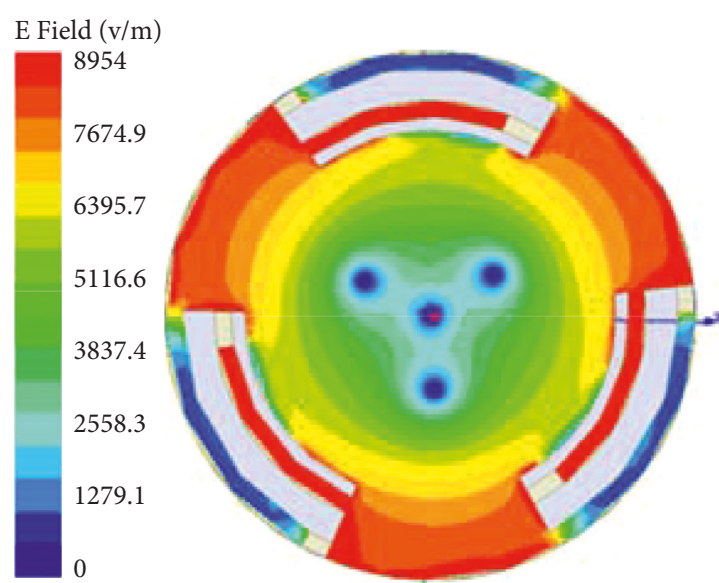

(a)

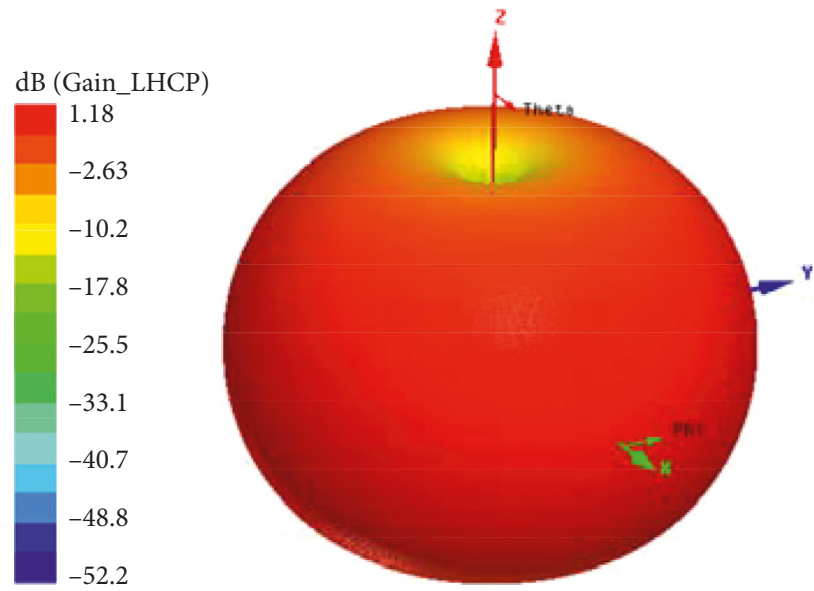

(c)

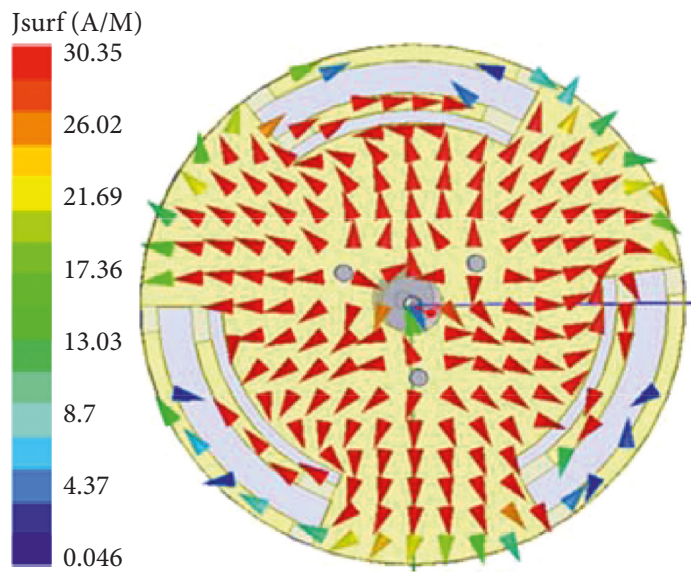

(b)

dB (Gain_RHCP)
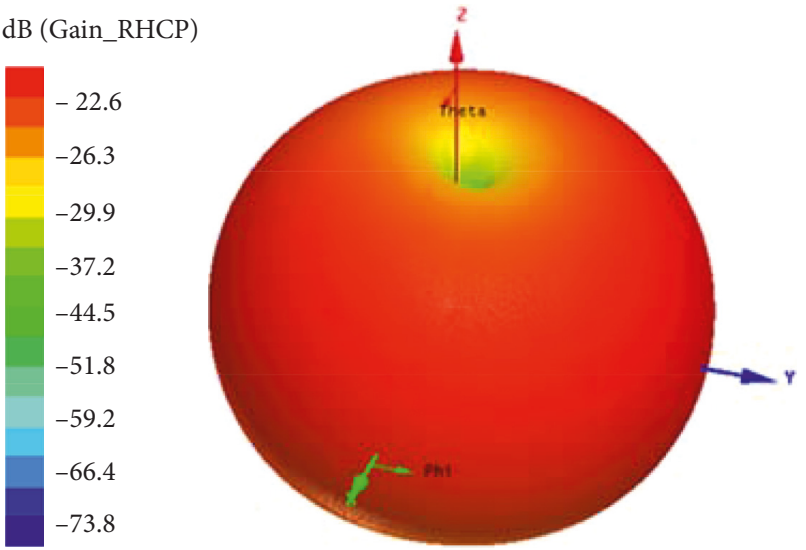

(d)

Figure 4: Energy and surface current distribution (a, b); radiation pattern plot gain LHCP (c) and gain RHCP (d).

3.2. Effect of Different $\varphi_{m}$. The size of each unit cavity is determined by $\varphi_{m}$. The length of the annular magnetic current source is controlled by the change of $\varphi_{m}$, whose effect is the same as that of the parameter $l_{m}$ shown in Figure 2(a). With the increase of $\varphi_{m}$, the resonant frequency is gradually reduced as can be seen from Figure 6(a) because the path of the equivalent magnetic current source is enlarged. When $\varphi_{m}=52 \mathrm{deg}$, the antenna can obtain the best resonance of centre frequency. Besides, the best axial ratio bandwidth is achieved at the same time shown in Figure 6(b).

\subsection{Effect of Different Diameter $d$ and the Location of Shorted} Aperture $R_{f}$. The shorted aperture plays a key role in antenna performance. It can be seen from Figure 7 that when the diameter of the shorting aperture is $1 \mathrm{~mm}$, a good match can be obtained at centre frequency. Meanwhile, the location of shorted aperture also has a significant effect on the resonant frequency. In Figure 8(a), it is shown that the resonant frequency increases with $R_{f}$. It is widely acknowledged that the fundamental mode $\mathrm{TM}_{01}$ of the circular patch can be realized by a central coaxial probe feed. The resonant frequency can be reduced clearly owing to the introduction of shorted aperture. The surface electric current on the patch is flowing along the radial direction. The shorted aperture plays the role of an electric wall, so the $\lambda / 4$ shorted wall is reduced in the mirror principle with the increase in $R_{f}$. However, the change of $R_{f}$ has negligible effect on the performance of $\mathrm{AR}$ as shown in Figure 8(b), thanks to the CSC.

\section{Result Analysis}

The proposed antenna is constructed by printed circuit board (PCB) technology and shorting pins are achieved by metallized holes. The antenna measurement was done in an anechoic chamber with a vector network analyzer. Firstly, the test distance should meet the far-field condition $\left(2 \times D^{2} / \lambda_{r}, D\right.$ is the maximum diameter of the antenna), and secondly, the tooling should be wrapped with absorbing material to prevent the influence on the antenna performance. Figures 9(a) and 9(b) display the S11 and AR result curves for the proposed CSC antenna. Both the measured and simulated results have good resonance at centre frequency. The resonance frequency is related to the size of some parameters of this antenna rigidly, and there is only one resonance region of this antenna. Therefore, the 


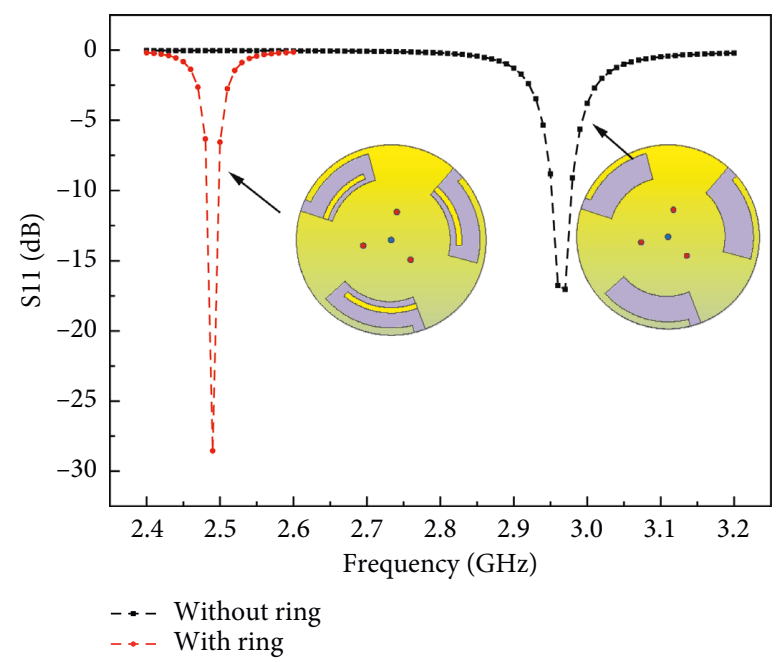

FIGURE 5: Comparison of with and without coupling ring.

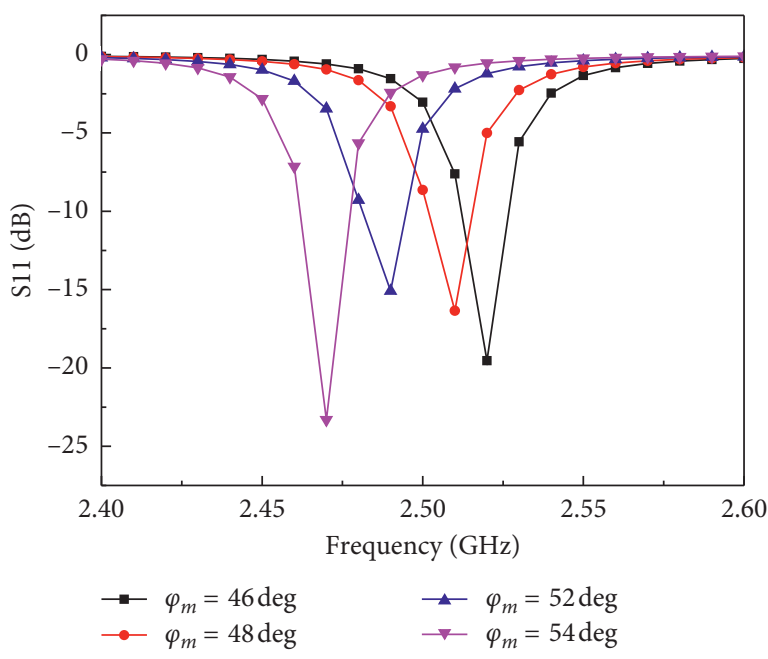

(a)

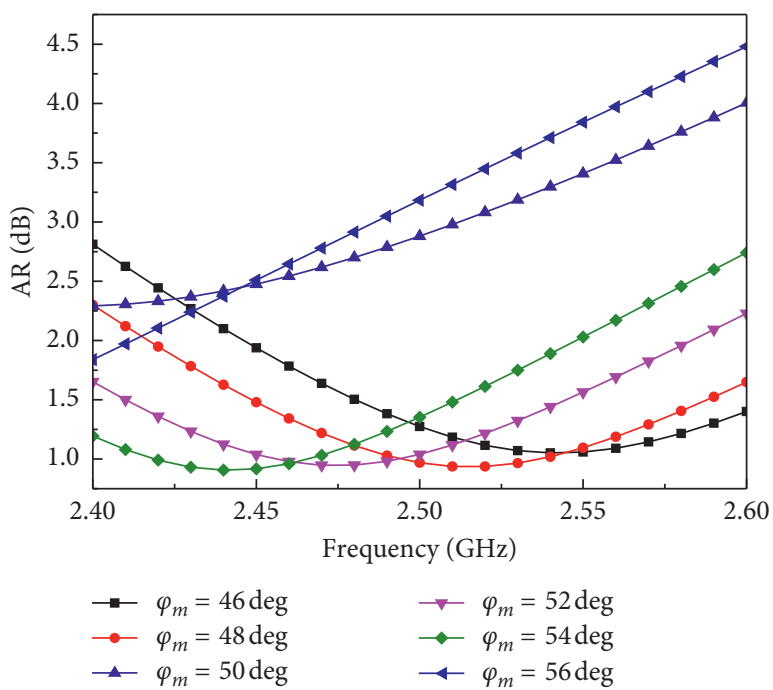

(b)

FIGURE 6: Effect of different $\varphi_{m}$ on antenna's performance: (a) S11 and (b) AR.

operation frequency band of $\mathrm{S} 11$ is limited (2481$2506 \mathrm{MHz}$ ). It can draw such a conclusion that the resonant frequency is especially sensitive to some parameters of the proposed antenna, such as the radius of the structure and the length of the double arcs. On the contrary, it has little effect on the uniform amplitude and the phase differences between the complementary dipoles. Hence, the frequency bandwidth of $A R$ is wide enough due to the CSC shown in Figure $9(\mathrm{~d})$.

The gain curve of this antenna is shown in Figure 9(c), and the peak gain occurs at the resonance frequency. Since the radiation from the nonazimuthal plane is caused by surface waves, the peak gain of the proposed antenna is slightly smaller. In order to enhance the gain of the proposed antenna, we can employ the following methods to improve the follow-up work. By introducing metallic reflectors on both sides of the antenna, the radiation energy is concentrated on the azimuth plane, and thus, the gain is improved effectively. Besides, similar to the Yagi-Uda antenna, several metallic rings are placed at the position that a quarter waveguide wavelength away from the antenna edge, guiding the radiation energy to the azimuth plane.

Similarly, the effect of the maximum gain and the lowest axial ratio can be obtained at the centre frequency shown in Figures 9(c) and 9(d). This indicates that good performance is achieved in the resonant region of the proposed antenna, which was constructed by printed circuit board (PCB) technique, whose prototype is shown in Figure 10.

The comparison between simulated and measured results of this antenna is shown in Figure 11. It can be seen from the figure that the antenna achieves omnidirectional radiation and satisfies the minimum nonroundness. The $2 \mathrm{D}$ pattern of the proposed antenna in the xoz plane is shown in Figure 11(b). Obviously, the two main lobe beam widths of 


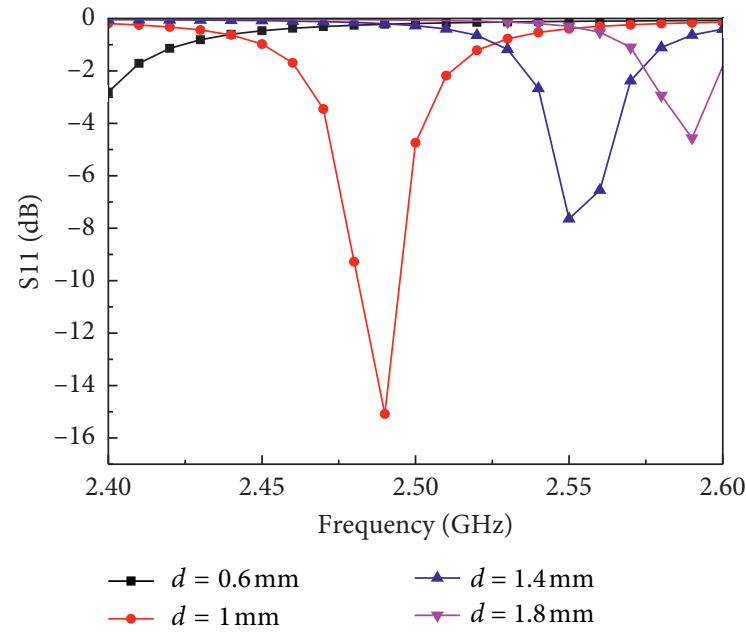

(a)

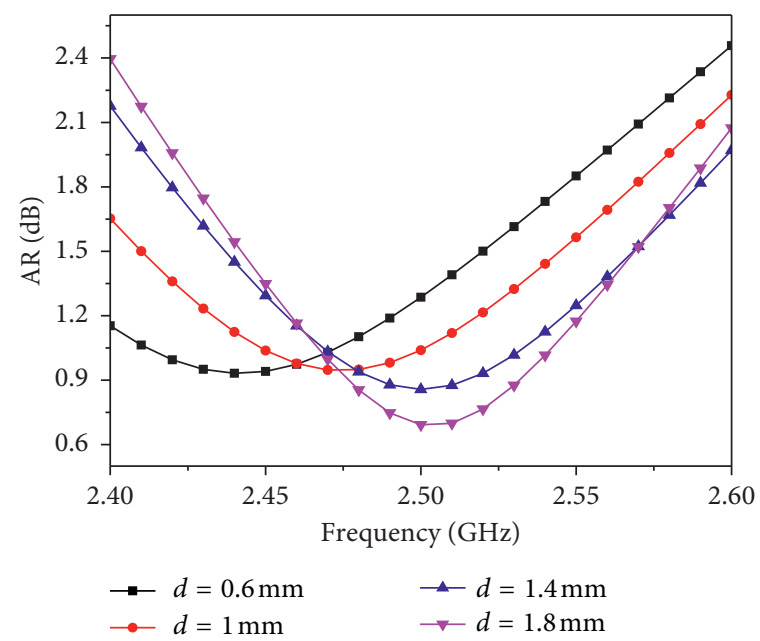

(b)

FIGURE 7: Effect of different $d$ on antenna's performance: (a) S11 and (b) AR.

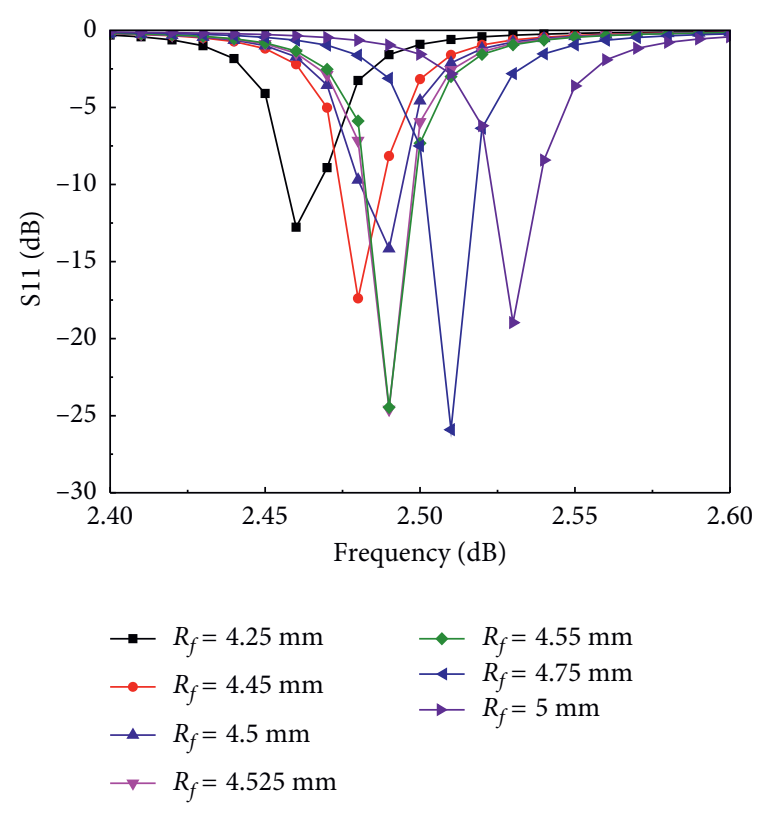

(a)

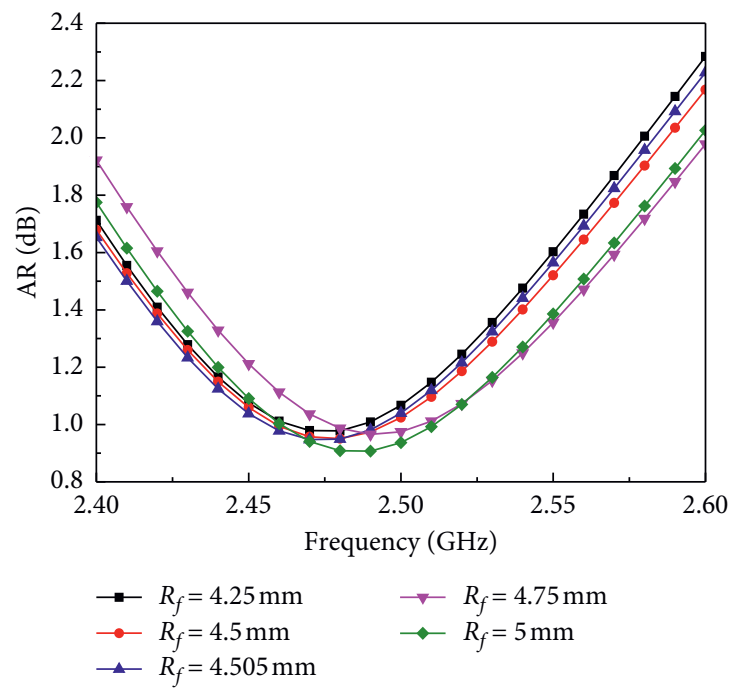

(b)

Figure 8: Effect of different $R_{f}$ on antenna's performance: (a) S11 and (b) AR.

the radiation pattern are relatively large, which is the direct reason for the low peak gain of the antenna. The measured results are perfectly in accordance with that of the simulation.

In this paper, an LHCP antenna is proposed. It can also be clearly seen from Figure 11 that the amplitude difference between the copolarization (LHCP) and cross-polarization (RHCP) is more than $20 \mathrm{~dB}$ in the same plane. The orientation of the electric field and magnetic field in far field is parallel to the profile and the tangential direction of the circular patch antenna, respectively, and the magnetic current phase ahead of the electric current phase. Using the right-hand theorem, the thumb points to the propagation direction, and the field phase leading component turns to the field phase lagging component. RHCP conforms to the righthand theorem, while LHCP corresponds to the left hand. That is to say, if the metallic current loop is connected to the upper right corner of the fan-shaped structure (opposite to the direction placed in this paper), an RHCP will be generated.

The proposed antenna has good AR characteristics in the whole azimuth plane as shown in Figure 9(b), thanks to the CSC. Moreover, the placement with different rotation angles of the proposed antenna has no impact on the overall performance. Since the proposed CSC antenna owns symmetrical structure to $z$-axis exactly, it is supposed that the AR 


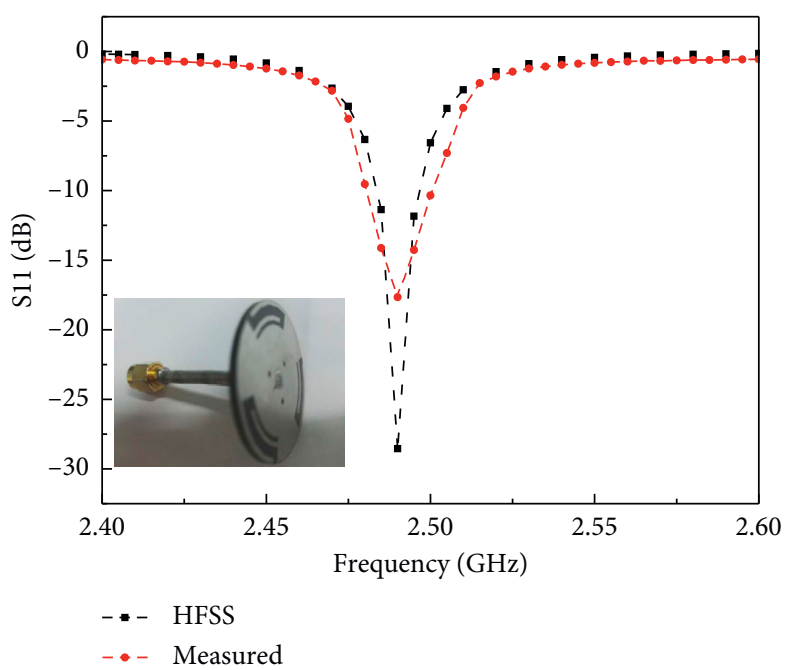

(a)

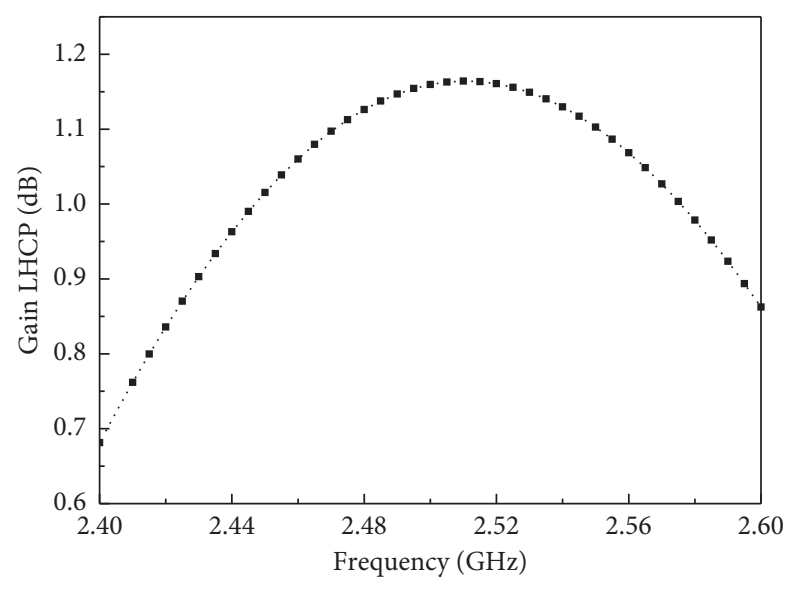

-.. Gain LHCP

(c)

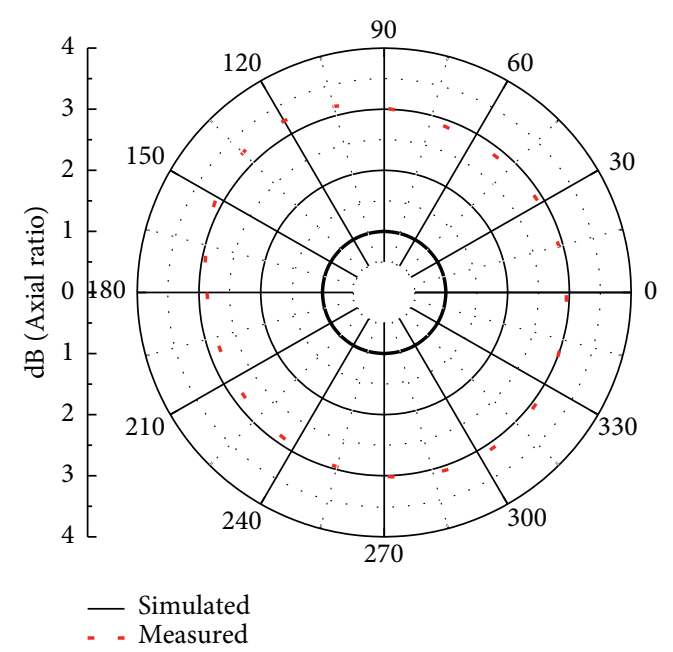

(b)

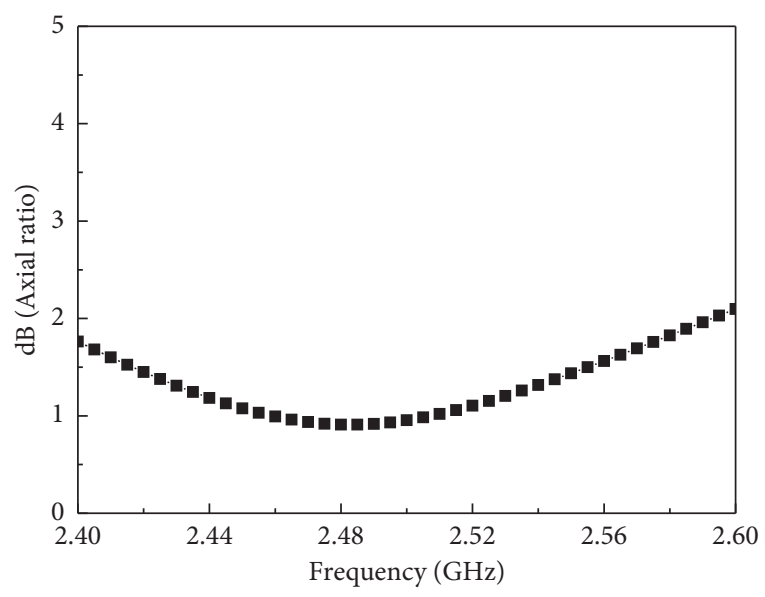

-... dB (Axial ratio)

Figure 9: $\mathrm{S} 11$ (a); AR curves of $\mathrm{fr}=2.4918 \mathrm{GHz}$ at $x o y$ plane (b); gain LHCP (c); axial ratio (d) of the proposed antenna.

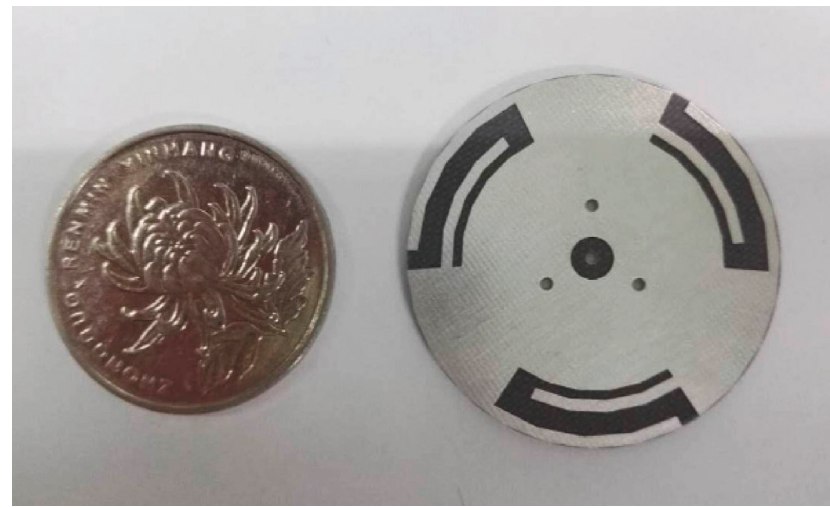

(a)

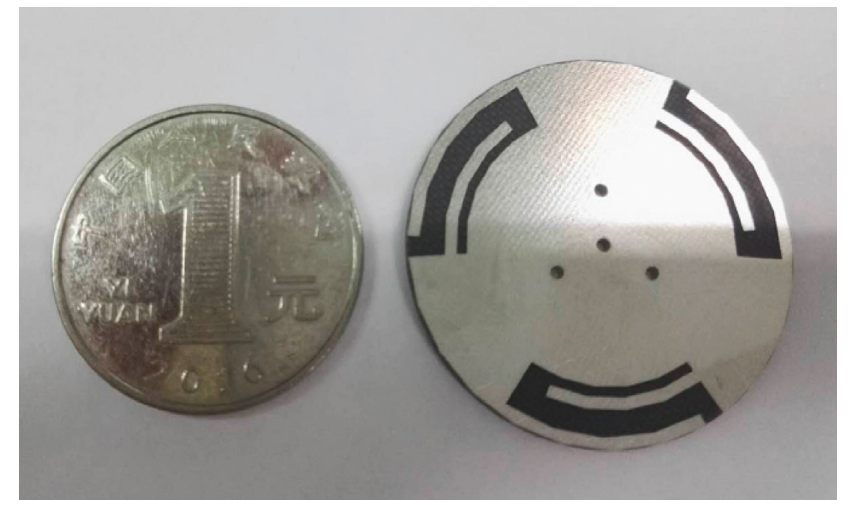

(b)

FIgURE 10: The proposed CP antenna bottom (a) and top (b).

and radiation patterns are similarly symmetrical to the $z$ axis. However, the measured $\mathrm{AR}$ and radiation pattern curves in Figures 9 and 11 show a little fluctuation. It is assumed that the discrepancies of the simulated and measured results are probably caused by the deviation of the measurements. The coaxial welding technology and 


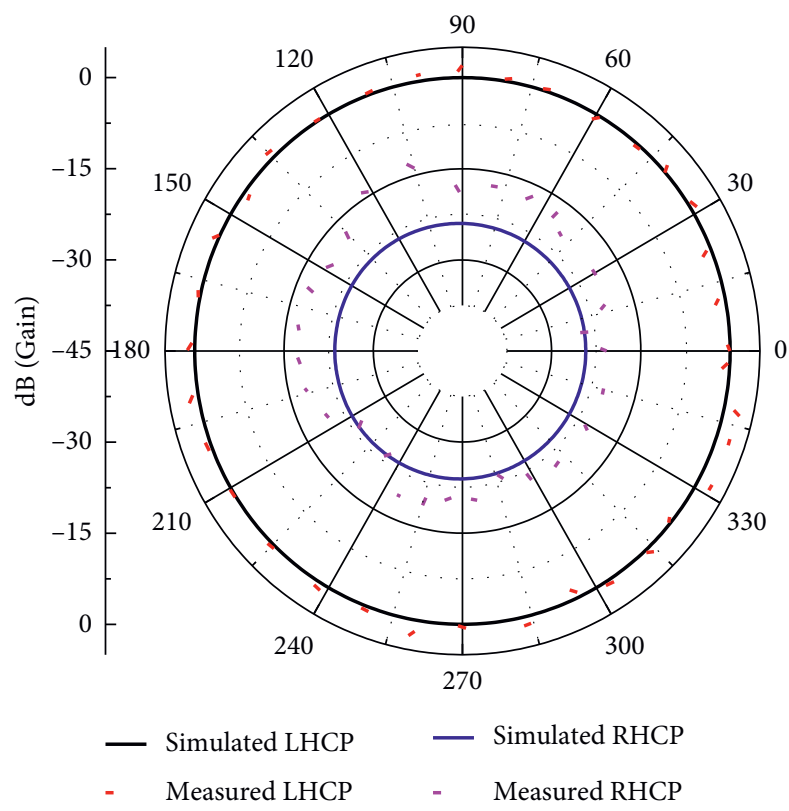

(a)

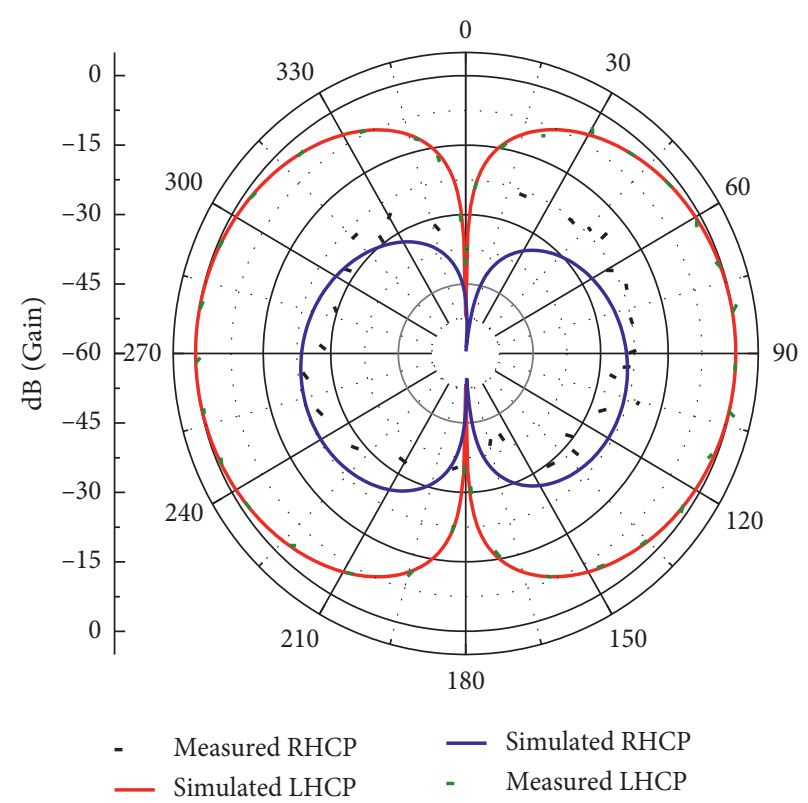

(b)

FiguRE 11: RHCP and LHCP patterns of fr $=2.49175 \mathrm{GHz}$; (a) normalized 2D pattern at $x o y$ plane; (b) normalized 2D pattern at $x o z$ plane.

TABLE 1: Comparison of reference and the proposed antenna

\begin{tabular}{lccccc}
\hline Reference & Structure & Usable bandwidth (\%) & Max gain $(\mathrm{dBi})$ & Dimensions $\left(\lambda_{0}\right.$ at centre frequency) & Height \\
\hline$[6]$ & Planar & 3.97 & 1.77 & $0.62 \lambda_{0} \times 0.62 \lambda_{0}$ & $0.029 \lambda_{0}$ \\
{$[2]$} & Planar & 4.3 & 3.3 & $1.74 \lambda_{0} \times 0.31 \lambda_{0}$ & $0.039 \lambda_{0}$ \\
{$[7]$} & Nonplanar & 0.9 & 1.01 & $0.16 \lambda_{0} \times 0.16 \lambda_{0}$ \\
{$[4]$} & Planar & 0.54 & -0.4 & $0.29 \lambda_{0} \times 0.29 \lambda_{0}$ \\
{$[5]$} & Nonplanar & 11.55 & 0.86 & $0.25 \lambda_{0} \times 0.25 \lambda_{0}$ & $0.016 \lambda_{0}$ \\
{$[11]$} & Planar & 0.78 & 0.82 & $0.29 \lambda_{0} \times 0.29 \lambda_{0}$ & $0.082 \lambda_{0}$ \\
{$[1]$} & Nonplanar & 7.3 & 2.04 & $0.32 \lambda_{0} \times 0.32 \lambda_{0}$ & $0.02 \lambda_{0}$ \\
This study & Planar & 1 & 1.15 & $0.27 \lambda_{0} \times 0.27 \lambda_{0}$ & $0.27 \lambda_{0}$ \\
\hline
\end{tabular}

measurement environment will have an unpredictable effect on the antenna performance. In general, the results of measurement and simulation precisely correspond with each other, which verify the design concept. The comparison between the proposed and reference antennas is shown in Table 1.

As can be seen from Table 1, the proposed antenna has the lowest profile among the reference antennas. Compared with several of the planar structures, such as $[2,6]$, they are larger in usable bandwidth than ours. However, the proposed antenna has reduced largely in size. More importantly, this proposed antenna possesses wider bandwidth and smaller size compared with the other planar structures. Finally, compared to nonplanar structures, the proposed antenna has the advantages of simple configuration, light weight, and low profile.

\section{Conclusion}

This paper presents a novel kind of CSC and omnidirectional $\mathrm{CP}$ antenna. It can easily be fabricated due to the simple construction. It is worth mentioning that the miniaturization technology of the proposed antenna is mainly realized by coupling arcs. Importantly, this work features the lowest profile and the smaller size relatively compared with the reference antennas. Moreover, it is omnidirectionally stable in the whole azimuth plane. The proposed antenna has an approximate $86 \%$ radiation efficiency, which has potential application in the field of the first generation of BeiDou Navigation System.

\section{Data Availability}

The data used to support the findings of this study are included within the article.

\section{Conflicts of Interest}

The authors declare that they have no conflicts of interest.

\section{Acknowledgments}

The authors wish to acknowledge the support from The National Science Foundation (61775175 and 61771378). 


\section{Supplementary Materials}

The data of different sheets are characterized by their own sheet names in the file of "graph-data.xlsx." For example, the sheet of "without-with-ring" is the comparison of the S11 under the different conditions of with or without the coupling ring. The sheet of "S11-d" is the S11 variation with different sizes of parameter "d." In addition, the value of several key parameters is given in the file of "size.docx." (Supplementary Materials)

\section{References}

[1] Y. M. Pan, K. W. Leung, and K. Lu, "Omnidirectional linearly and circularly polarized rectangular dielectric resonator antennas," IEEE Transactions on Antennas and Propagation, vol. 60, no. 2, pp. 751-759, 2012.

[2] J. Liu, Y. Li, Z. Liang, and Y. Long, "A planar quasimagnetic-electric circularly polarized antenna," IEEE Transactions on Antennas and Propagation, vol. 64, no. 6, pp. 2108-2114, 2016.

[3] Y. Yu, Z. Shen, and S. He, "Compact omnidirectional antenna of circular polarization," IEEE Antennas and Wireless Propagation Letters, vol. 11, pp. 1466-1469, 2012.

[4] B.-C. Park and J.-H. Lee, "Omnidirectional circularly polarized antenna utilizing zeroth-order resonance of epsilon negative transmission line," IEEE Transactions on Antennas and Propagation, vol. 59, no. 7, pp. 2717-2721, 2011.

[5] B. Li, S.-W. Liao, and Q. Xue, "Omnidirectional circularly polarized antenna combining monopole and loop radiators," IEEE Antennas and Wireless Propagation Letters, vol. 12, pp. 607-610, 2013.

[6] Q.-X. Chu, M. Ye, and X.-R. Li, "A low-profile omnidirectional circularly polarized antenna using planar sector-shaped endfire elements," IEEE Transactions on Antennas and Propagation, vol. 65, no. 5, pp. 2240-2247, 2017.

[7] D. Wu, X. Chen, L. Yang, G. Fu, and X. Shi, "Compact and low-profile omnidirectional circularly polarized antenna with four coupling arcs for UAV applications," IEEE Antennas and Wireless Propagation Letters, vol. 16, pp. 2919-2922, 2017.

[8] Y. M. Pan, S. Y. Zheng, and B. J. Hu, "Wideband and lowprofile omnidirectional circularly polarized patch antenna," IEEE Transactions on Antennas and Propagation, vol. 62, no. 8, pp. 4347-4351, 2014.

[9] J. Liu, X. Quan, H. Wong, H. W. Lai, and Y. Long, "Design and analysis of a low-profile and broadband microstrip monopolar patch antenna," IEEE Transactions on Antennas and Propagation, vol. 61, no. 1, pp. 11-18, 2013.

[10] B.-C. Park and J.-H. Lee, "Dual-band omnidirectional circularly polarized antenna using zeroth- and first-order modes," IEEE Antennas and Wireless Propagation Letters, vol. 11, pp. 407-410, 2012.

[11] L. Peng, K. Sun, X. Jiang, S.-M. Li, and C.-L. Ruan, "EZR-MZR resonators for compact low-profile omnidirectional circularpolarized antenna design," IEEE Photonics Journal Article Sequence, vol. 9, no. 4, pp. 1-15, 2017. 


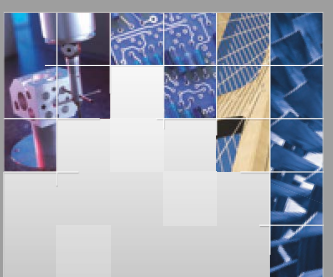

\section{Enfincering}
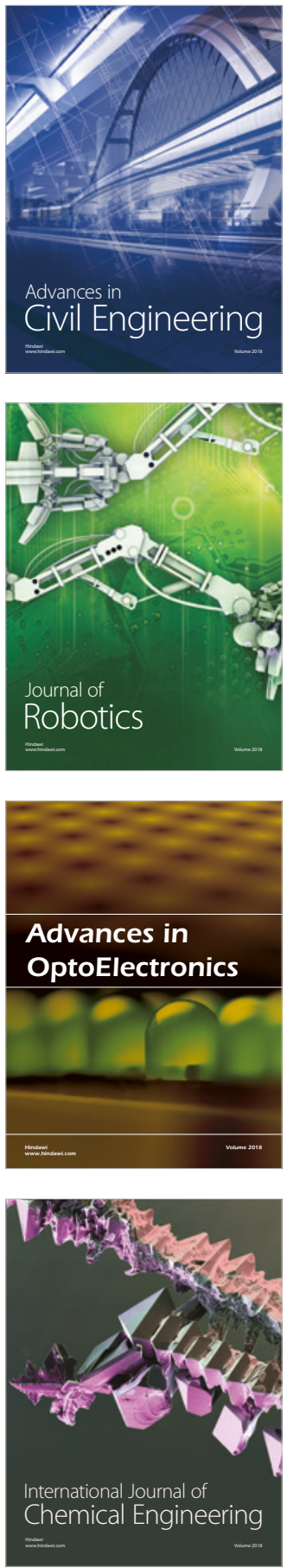

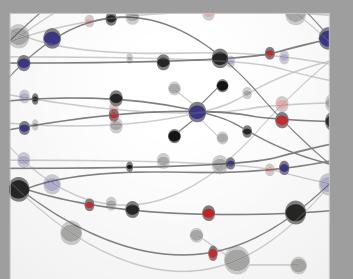

\section{Rotating \\ Machinery}

The Scientific World Journal

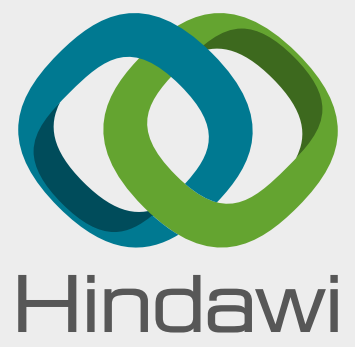

Submit your manuscripts at

www.hindawi.com
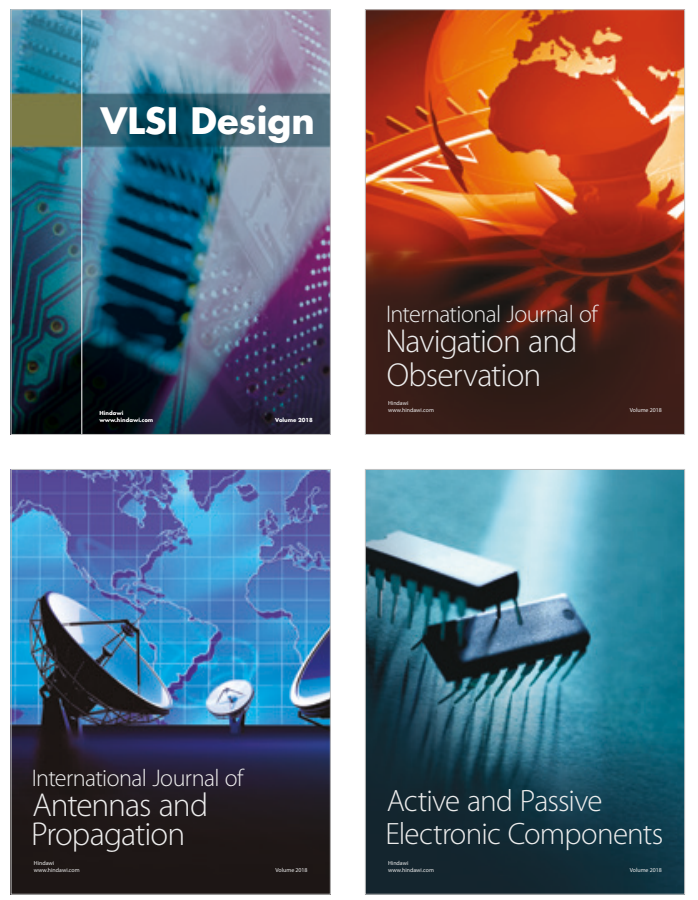
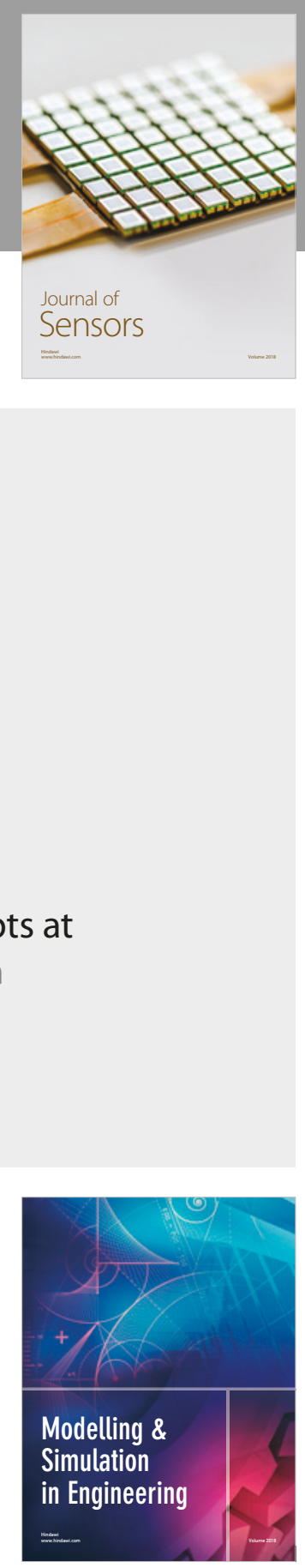

\section{Advances \\ Multimedia}
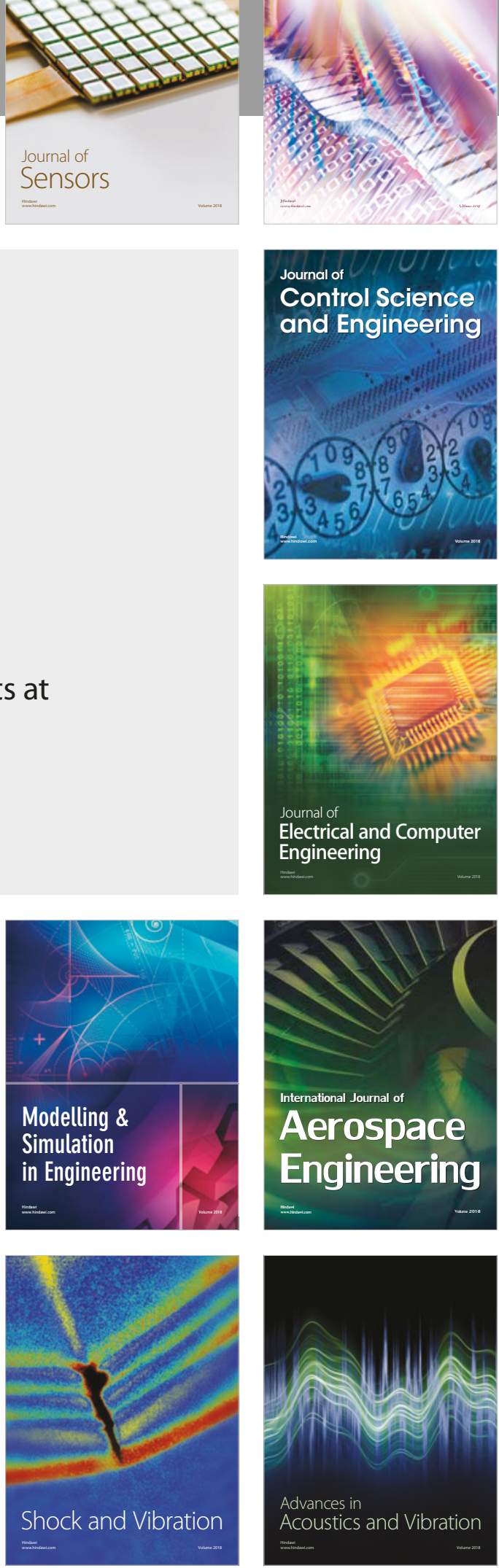